\title{
Una aproximación a las valoraciones en torno a la discriminación de género en tres medios de la prensa chilena
}

\author{
Silvana Guerrero González y Valeska Muller González* \\ Pontificia Universidad Católica de Chile
}

\begin{abstract}
Resumen
En esta investigación se analiza, desde el punto de vista cualitativo, el modo cómo tres medios de prensa escrita nacional, representada por El Mercurio, La Nación y El Mostrador, construyen la representación de las valoraciones sobre el tema de la discriminación de género a propósito del caso Zamudio y la Ley Antidiscriminación, a la luz de la Teoría de la Valoración (White 2004; Martin 2005; Martin y White 2005; y Martin y Rose 2007; Hood 2010; Kaplan 2004; Oteíza 2006; Oteíza y Pinuer 2012). Para tal efecto, se seleccionaron seis crónicas noticiosas aparecidas en el marco de la noticia sobre la golpiza a Daniel Zamudio, en las que fue de gran importancia analizar cómo se presentaron las identidades de un nosotros no homosexual y contrario a esta tendencia sexual, frente a un ellos homosexual y/o defensor
\end{abstract}

* Para correspondencia, dirigirse a: Silvana Guerrero González (smguerrero@uc.cl), Pontificia Universidad Católica de Chile, Facultad de Letras, Departamento de Ciencias del Lenguaje, Campus San Joaquín / Av. Vicuña Mackenna 4860, Santiago de Chile, o a Valeska Muller González (vmuller@uc.cl), Pontificia Universidad Católica de Chile, Campus Oriente, Av. Jaime Guzmán E. 3300, Providencia, Santiago de Chile. 
de la homosexualidad. Los hallazgos de la investigación dieron cuenta de la relevancia de las valoraciones en la prensa chilena. El Mercurio se confirma como un medio de tendencia conservadora dirigido a un nivel sociocultural alto, preocupado por las decisiones que regulan la vida en sociedad. La Nación se propone como el canal del Gobierno, ocupado en valorar las acciones del Gobierno anterior y actual. Por último, El Mostrador se posiciona como un medio más combativo, donde se transmite sin censura la discusión entre los diferentes actores sociales, que tienen cabida en este medio. En consecuencia, se trata aquí de una práctica social -el discurso de los medios de comunicación- cuyo poder es generalmente simbólico y persuasivo, donde se despliega una gama de recursos propios de los sistemas semánticos analizados.

Palabras clave: Teoría de la Valoración (TVA), discriminación de género, medios de prensa.

\title{
AN APPROACH TO THE APPRAISAL OF GENDER DISCRIMINATION IN THREE CHILEAN NEWSPAPERS
}

\begin{abstract}
This research study analyzes, from a qualitative point of view, the manner in wich three national newspapers -El Mercurio, La Nación and El Mostrador-build the evaluation representation on the subject of gender discrimination within the context of the Zamudio case and the Antidiscrimination Law, in light of the Appraisal Theory (White 2004; Martin 2005; Martin y White 2005; Martin y Rose 2007; Hood 2010; Kaplan 2004; Oteíza 2006; Oteíza and Merino 2012). To that effect, three stories on the attack on Daniel Zamudio were analyzed, primarily focusing on the manner in wich the identity of a non-gay $u s$, against this sexual orientation, is presented as opposed to a gay or gay-supporting them. The findings of this research account for the relevance of evaluations in the Chilean press. El Mercurio is confirmed as a conservative newpapers that aims at a high socio-cultural level, concerned with the decisions that regulate life in society. La Nación, in turn, establishes itself as the government channel, evaluating the actions of the current government and those of the former. Finally, El Mostrador positions itself as a more combatant medium, where the discussion among different social actors that belong to this newspapers is transmitted without censorship. Consequently, the discourse of the mass media constitutes a social practice whose power is generally symbolic and
\end{abstract}


persuasive, where a variety of resources belonging to the semantic systems analyzed is displayed.

Key words: Appraisal Theory, gender discrimination, newspapers.

Recibido: $16 / 08 / 2012 \quad$ Aceptado: $28 / 11 / 2012$

\section{INTRODUCCIÓN}

El siguiente estudio analiza el modo cómo tres diarios chilenos abordaron el tema de la discriminación de género a propósito del caso Zamudio y la Ley Antidiscriminación. El contexto de aparición de las crónicas noticiosas que conforman el corpus del estudio corresponde al periodo anterior a la aprobación definitiva de la Ley Antidiscriminación en el Congreso Nacional, e inmediatamente siguiente a la golpiza y posterior muerte del joven Daniel Zamudio, quien fue victimizado por su orientación sexual, en marzo de 2012 en la ciudad de Santiago de Chile. Esta situación tiene que ver con la heterogeneidad de los grupos sociales; diferencias de religiones, de razas, de clases sociales o nivel cultural, de ideología política e, incluso, de tendencias sexuales. Todas ellas instalan la diversidad y se hace necesario, por tanto, normar la convivencia con nuevas leyes que protejan los derechos de todos los ciudadanos independientemente de su posición en la estructura social. En este escenario, una de las prácticas sociales donde se revelan con mayor fuerza las diferentes posturas y donde se construyen y reproducen ideologías a favor y en contra de la desigualdad y la discriminación, es el discurso de los medios de comunicación, foco de nuestro análisis.

El proyecto de ley que establece medidas contra la discriminación, del 14 de marzo de 2005, intenta revertir el hecho de que el tratamiento que se la ha dado a este tema en Chile, en materia de ley, resulta pobre y deficiente. A este respecto, solo existe la ley 19.733, que regula la Libertad de Opinión e Información y Ejercicio del Periodismo y que en su artículo 31 incorpora la incitación al odio como única mención a la violación del principio de no discriminación. El proyecto de ley en discusión contempla una "Norma Penal Especial" en virtud de la cual se propone que se incorpore a la enumeración que hace el artículo 12 del Código Penal de las circunstancias que agravan la responsabilidad criminal, una nueva agravante consistente en 
cometer el delito por una motivación discriminatoria fundada en la raza, color, origen étnico, edad, sexo, género, religión, creencia, opinión política o de otra índole, nacimiento, origen nacional, cultural o socioeconómico, idioma o lengua, estado civil, orientación sexual, enfermedad, discapacidad, estructura genética o cualquiera otra condición social o individual (Título3, Artículo 9) ${ }^{1}$.

El proyecto fue ingresado a la Cámara de Diputados el 22 de marzo de 2005 y desde entonces ha sido discutido y aprobado con modificaciones, por la Cámara y el Senado, sin llegar a su aprobación definitiva. En tanto, la noche del 2 de marzo de 2012 un hecho repudiable alertó a la opinión pública y puso a la clase política en cuestión por la lentitud en la aprobación de este proyecto. En efecto, el brutal ataque a Daniel Zamudio la citada noche produjo conmoción en la sociedad chilena y levantó el debate respecto a la homofobia en el país y a la falta de una ley antidiscriminación relacionada con este tipo de crímenes. Cuatro días después de los lamentables hechos, el Gobierno chileno pondría urgencia a la Ley Antidiscriminación y se iniciaría una serie de debates que fueron registrados por la prensa ${ }^{2}$. En definitiva, dada la importancia que reviste este tema para la sociedad y el hecho de que no ha sido tratado con el interés que se merece -prueba de ello es la falta de una legislación clara al respecto, como en otros países- y que incluso desde el punto de vista de los Estudios del Discurso en Latinoamérica tampoco ha recibido la suficiente atención (cf. Pilleux y Merino 2004; Merino 2007; Merino et al. 2008; Pino y Merino 2010; Collado y Jofré 2010; Merino y Tileaga 2011; Oteíza y Pinuer 2012), nos proponemos revisar y analizar críticamente seis crónicas aparecidas en tres diarios de la prensa chilena, con diferentes orientaciones políticas, a fin de aportar a los estudios sobre discriminación de género. Dichos diarios son los siguientes: El Mercurio, diario considerado conservador y de derecha; La Nación, diario de circulación digital, que podría considerarse de centro; y, El Mostrador, diario digital asociado a corrientes políticas de izquierda. En concreto, en esta investigación se intentará determinar el modo cómo tres medios de prensa nacional construyen la representación intersubjetiva de las valoraciones en relación con el tema de la discriminación de género a propósito del caso Zamudio y la Ley Antidiscriminación.

\footnotetext{
Disponible en el sitio Web de la Cámara de Diputados de Chile ( www.camara.cl).

2 En el transcurso del desarrollo de esta investigación fue aprobado el proyecto de Ley Antidiscriminación.
} 
El marco teórico y metodológico de esta investigación considera el ámbito de los Estudios Críticos del Discurso y dentro de este, los aportes de la teoría de Fairclough $(1995,2008)$ y de van Dijk (2009). El análisis, en particular, está basado en los sistemas semánticos de la Teoría de la Valoración (en adelante, TAV, estudiada, entre otros, por White 2004; Martin 2005; Martin y White 2005; y Martin y Rose 2007; Hood 2010; Kaplan 2004; Oteíza 2006; Oteíza y Pinuer 2012).

\section{FUNDAMENTOS TEÓRICO METODOLÓGICOS}

\subsection{Estudios Críticos del Discurso}

Este trabajo se enmarca en los Estudios Críticos del Discurso (en adelante, ECD $)^{3}$. Respecto de esto, recordemos que el objetivo que se ha planteado para el Análisis Crítico del Discurso (ACD) es, según Wodak (2003:19):

analizar, ya sean estas opacas o transparentes, las relaciones de dominación, discriminación, poder y control, tal como se manifiestan a través del lenguaje. En otras palabras, el ACD se propone investigar de forma crítica la desigualad social tal como viene expresada, señalada, constituida, legitimada, etcétera, por los usos del lenguaje (es decir por el discurso).

De acuerdo con estos presupuestos generales, en los siguientes apartados desarrollaremos los aportes teóricos más relevantes para los objetivos de nuestra investigación, enfocándonos en aquellos antecedentes que nos permitan determinar, de acuerdo con van Dijk (2009), cómo el poder funciona como abuso de poder y cómo se ejerce dominación desde los discursos, generando y legitimando diversas formas de desigualdad social. En otras palabras: "los grupos dominantes son los que tienen acceso a la manipulación y al uso de estructuras discursivas de dominación, de desigualdad y de

\footnotetext{
3 Mientras que la acostumbrada denominación de esta línea de investigación ha sido Análisis Crítico del Discurso, van Dijk (2009) ha comenzado a emplear un término más general, a saber, Estudios Críticos del Discurso (ECD), a fin de enfatizar que el estudio crítico no constituye un método de análisis, sino que conlleva dimensiones tanto teóricas como aplicadas. En consecuencia, en esta investigación optaremos por hablar de ECD en aquellos casos en que sea pertinente.
} 
limitaciones de la libertad" (van Dijk 1994: 8), toda vez que-según van Dijk (ibíd.)- el poder de los medios de comunicación es generalmente simbólico y persuasivo, en el sentido de que tiene la posibilidad de controlar, en mayor o en menor medida, la mente de los lectores. En consecuencia, el control de acciones, meta última del poder, se realiza de manera indirecta cuando se planea el control de intenciones, de proyectos, de conocimientos por alcanzar, de creencias o de opiniones.

\subsubsection{Estudios críticos del discurso y discriminación}

Fairclough (1995) postula que el discurso es un complejo de tres elementos interrelacionados: 1) el texto y sus elementos constitutivos, 2) la práctica discursiva que los hablantes realizan en los textos y 3 ) la práctica social donde tanto la práctica discursiva como el texto son modelados ideológicamente ${ }^{4}$. Con base en este planteamiento y de acuerdo con los objetivos de esta investigación, las prácticas discusivas que serán objeto de estudio pueden considerarse ideológicamente en la medida en que contribuyen a hacer o deshacer realidades sociales.

Uno de los investigadores más preocupados por el ECD en relación con la discriminación es van Dijk (2003a, 2003b, 2004a, 2004b, 2007, 2008a). Este autor señala -en relación con la ideología en tanto fundamento de las prácticas sociales- que las ideologías constituyen sistemas de grupos sociales y movimientos y que, por tanto, no solo dan sentido al mundo, sino que también fundamentan las prácticas sociales de sus miembros. En consecuencia, "las ideologías sexistas o racistas son la base de la discriminación" (van Dijk 2003a:16). Lo central en esta perspectiva es que las ideologías sitúan a un Nosotros contra un Ellos, debido a que son creencias compartidas socialmente y se vinculan a las propiedades características de un grupo, como la identidad o los intereses.

4 Fairclough (1995) retoma y reformula la noción de órdenes del discurso de Foucault (1992[1970]). Foucault señala que todo discurso existe en el contexto de un sistema de regulaciones (rarefacción), por tanto, la producción de discursos es la rarefacción de los mismos. De acuerdo con Fairclough (2008), el orden del discurso es el conjunto ordenado de prácticas discursivas asociadas con un dominio o institución social particular, esto es, las convenciones que subyacen a los eventos discursivos corresponden a los órdenes del discurso. "El orden del discurso de algunos dominios sociales es la totalidad de sus prácticas discursivas, y las relaciones (de complementariedad, inclusión/exclusión, oposición) entre ellas [...] Y el orden del discurso de una sociedad es el conjunto de estos órdenes del discurso más 'locales', y las relaciones entre ellos [...] Los límites y separaciones entre, y dentro de los órdenes del discurso, pueden ser puntos de conflicto y de disputas (Bernstein 1990), que pueden debilitarse o fortalecerse, como parte de conflictos y luchas sociales más amplios" (p. 173). 
Dentro de los temas más abordados en la relación entre ECD y discriminación se encuentra el racismo. Según Traverso-Yépez (2005) -siguiendo a Wieviorcka (1992) - el racismo es un término demasiado amplio, pues incluye diversas expresiones de segregación, rechazo, exclusión y odio en función de la dificultad de asumir al otro (diferente) en su legitimidad como ser humano. Asimismo, plantea que las ideas y las prácticas racistas, son difíciles de ser reconocidas como tales, cuando son parte de lo cotidiano y, en consecuencia, se vuelven automatismos con los cuales se actúa irreflexivamente. Al respecto, Rodríguez-Bailón y Moya (2003) dan cuenta de que pueden existir formas encubiertas de discriminación que conllevan actitudes antagónicas en los individuos quienes, de hecho, no siempre son conscientes de ellas. En esta línea, es el mismo van Dijk (2007) quien postula dos formas de discurso racista, a saber, el dirigido al otro diferente y el sobre el otro diferente. Ambas perspectivas son útiles a la hora de analizar el modo cómo los diferentes actores se dirigen a Daniel Zamudio, considerado el otro diferente, y convierten en tema su golpiza y posterior muerte ${ }^{5}$. Además de estos antecedentes, otro concepto ampliamente desarrollado es el de prejuicio en relación con los estereotipos y la discriminación. Merino et al. (2008) lo describen de la siguiente manera respecto de los mapuches:

[...] el prejuicio constituye el componente más afectivo del rechazo grupal en tanto los estereotipos constituyen el componente más cognitivo del rechazo grupal. El prejuicio es entendido como una actitud negativa o una predisposición a adoptar un comportamiento negativo hacia un grupo [...] Los estereotipos y el prejuicio generalmente desembocan en problemas sociales $[\ldots]$ Uno de los problemas sociales generados por el prejuicio es la discriminación entendida como cualquier conducta desplegada por miembros de endogrupos que otorga un tratamiento injusto y desigual a los miembros de exogrupos en razón de su mera pertenencia al exogrupo (Allport 1954). La discriminación está basada, entonces, en una distinción de categorías naturales o sociales que no

\footnotetext{
5 De acuerdo con van Leeuwen (1996), entre las estrategias para la representación de los actores sociales, están: la individualización, esto es, la representación determinada o diferenciada de un actor social es aquella en la que se explicita sus diferencias como individuo o como miembro de un grupo social específico, creando así la diferencia entre "yo" y el "otro", o entre "nosotros" y "ellos"; y, la identificación, es decir, cuando el actor es definido en términos de una esencia más o menos permanente de algo que los constituye y que no puede eludir, entre otras estrategias que serán útiles para la discusión de esta investigación.
} 
guardan ninguna relación con los méritos, capacidades o acciones concretas de los miembros específicos de esas categorías (282).

También en relación con los mapuches, Pilleux y Merino (2004) enfatizan que los prejuicios y actitudes racistas son los que, finalmente, llevan a la discriminación. En consecuencia, "la exclusión social que vivencian los miembros de grupos minoritarios en las sociedades dominantes es una problemática que genera situaciones de menoscabo para el grupo dominado" (Pino y Merino 2010: 105-106). De igual forma, de la Fuente (2004) en su estudio sobre racismo hacia inmigrantes plantea que es innegable que el concepto de raza existe como construcción social, dado que sobre él se discrimina y se margina continuamente a multitud de personas, por tanto, dicho concepto puede tener validez como concepto sociológico pero no como término biológico. Estos antecedentes son, asimismo, válidos para pensar en los homosexuales como una minoría, cuestión fundamental cuando esta investigación parte de un caso concreto: el de Daniel Zamudio.

Finalmente, para nuestros propósitos, consideramos necesario enfatizar en lo que Soto (2011, reseñando a van Dijk 2009) precisa en relación con el discurso: "concebido como estructura verbal, como medio de comunicación de creencias y como forma de acción e interacción en situaciones sociales, el discurso desempeña un papel privilegiado en la interacción que los sujetos tienen entre sí" (435). Además, señala el autor, el hablante puede organizar el discurso de manera tal de favorecer sus perspectivas sobre la situación que comunica y desfavorecer las perspectivas contrarias. Así, la utilización de determinados recursos lingüísticos (por ejemplo, la selección léxica o la información que porta la cláusula -cantidad de complementos, explicitación del agente, entre otros aspectos-) parece estar al servicio de este objetivo.

\subsubsection{Metodologías en Estudios críticos del discurso y discriminación}

El interés del ACD "parte del hecho de que los grupos sociales dominantes ejercen su poder a través de medios económicos o legales pero también a través de medios lingüísticos, la utilización de un tipo de lenguaje concreto puede ser un símbolo de poder" (de la Fuente, 2004: 2). En sus estudios sobre racismo, van Dijk ha empleado diversas metodologías, determinadas, por supuesto, por los requerimientos implicados en los distintos corpus de trabajo. La conversación cotidiana, los debates parlamentarios, los textos escolares y, sobre todo, la prensa han constituido algunos de sus principales corpus de trabajo, pues en ellos se habla/escribe sobre las minorías, sobre los inmigrantes y sobre los refugiados. En concreto, se ha analizado el control 
sobre la entonación, el control sobre las estructuras de tópico, el control de superestructuras y el control sobre estructuras semánticas locales, entre otros aspectos del discurso. A través del análisis de los textos, el autor pretende, grosso modo, saber cómo piensan y qué actitud tienen y qué prejuicios existen en las personas cuando hablan sobre las minorías, o bien, dilucidar cómo se producen y reproducen los prejuicios, las ideas racistas y discriminatorias en la sociedad. En otros estudios en el marco de la discriminación, mediante entrevistas semi-estructuradas, se han obtenido y analizado los datos respecto de la percepción que los mapuches tienen acerca de la discriminación hacia su etnia en el contexto de las estrategias discursivas semánticas (Pilleux y Merino 2004, Merino 2007, Merino et al. 2008, Pino y Merino 2010). De la Fuente (2004) utiliza como corpus los medios de comunicación para analizar el racismo, y Soler (2004) el discurso de y sobre la escuela, a fin de estudiar la representación de indígenas en los manuales escolares de ciencias sociales en Colombia.

Los anteriores son solo algunos de los recursos metodológicos y ámbitos de prácticas discursivas con los que se ha investigado la discriminación en los ECD. En relación con nuestro objeto de estudio -la prensa- la investigación se ha centrado en "¿cómo se hacen y se producen las noticias? ¿de dónde vienen? ¿cuál es el trabajo cotidiano del periodista?" (van Dijk, ibíd.: 39). Considerando que los medios masivos, en general, son cruciales para la comunicación de ideologías raciales o étnicas hacia y entre la mayoría del público (Hartmann \& Husband 1974; Wilson \& Gutiérrez 1985, cit. en van Dijk 1988), a través de un análisis desde la Teoría de la valoración (White 2004; Martin 2005; Martin y White 2005; y Martin y Rose 2007; Hood 2010; Kaplan 2004; Oteíza 2006; Oteíza y Pinuer 2012), indagaremos el modo cómo se construye la representación intersubjetiva de las valoraciones en el discurso de tres medios de prensa nacional, en relación con el tema de la discriminación de género a propósito del caso Zamudio y la Ley Antidiscriminación.

Finalmente, consideramos necesario explicitar que de acuerdo con las referencias teórico metodológicas antes mencionadas, en esta investigación se vincularán directamente los antecedentes de los ECD, los estudios sobre discriminación y la TAV, puesto que el análisis se centra en la discriminación de género utilizando las herramientas que proporciona la TAV, lo que nos permitirá construir, finalmente, un perfil ideológico de los tres medios de prensa analizados, considerando los presupuestos que utiliza van Dijk (2003a, 2003b, 2004a, 2004b, 2007, 2008a, 2009). 


\subsection{Metodología de ANÁLISIS}

\subsubsection{Corpus}

El corpus de nuestra investigación está constituido por 6 crónicas noticiosas aparecidas entre el 31 de marzo de 2012 y el 9 de abril de 2012 en el marco de la noticia sobre la golpiza a Daniel Zamudio y, en consecuencia, de la Ley Antidiscriminación. Cabe mencionar, además, que debido a que no es nuestro objetivo comparar de manera cuantitativa los recursos lingüísticos utilizados en los tres medios seleccionados, sino observar cómo distintos medios abordaron los hechos, las noticias elegidas no corresponden exactamente al mismo suceso, aunque comparten un marco general, ni tienen el mismo número de palabras, pues la selección obedece a la relevancia de las mismas. En este sentido, es necesario precisar que este estudio es de naturaleza cualitativa, e incluye dos fases: una descriptiva, donde se analizará el funcionamiento de los subsistemas de valoración en el corpus a través el análisis de la representación de objetos, actores y eventos sociales, y otra de carácter interpretativo, donde se intentará caracterizar ideológicamente a cada medio de prensa de acuerdo con el análisis realizado. A continuación, en el Cuadro 1, se indica el detalle del titular de la crónica, la fecha de aparición, el periódico y la tendencia política del periódico.

\section{Cuadro 1: Descripción del corpus}

\begin{tabular}{|l|l|l|l|}
\hline \multicolumn{1}{|c|}{ Titular } & Fecha & Periódico & $\begin{array}{l}\text { Tendencia } \\
\text { política } \\
\text { periódico }\end{array}$ \\
\hline $\begin{array}{l}\text { Movilh acusa a Iglesia Católica y la } \\
\text { UDI de realizar "campaña del terror" } \\
\text { en contra de ley antidiscriminación }\end{array}$ & $\begin{array}{l}31 \mathrm{de} \\
\text { marzo }\end{array}$ & El Mostrador & Izquierda \\
\hline $\begin{array}{l}\text { La Moneda desestima críticas del } \\
\text { Movilh a Ley Antidiscriminación }\end{array}$ & $\begin{array}{l}3 \mathrm{de} \\
\text { abril }\end{array}$ & La Nación & Centro \\
\hline $\begin{array}{l}\text { Chadwick desestima que Ley } \\
\text { Antidiscriminación abra la puerta al } \\
\text { matrimonio homosexual }\end{array}$ & $\begin{array}{l}3 \mathrm{de} \\
\text { abril }\end{array}$ & El Mercurio & Derecha \\
\hline $\begin{array}{l}\text { Arzobispo Ezzati acusa al Movilh de } \\
\text { "aprovecharse" del caso Zamudio }\end{array}$ & $\begin{array}{l}4 \mathrm{de} \\
\text { abril }\end{array}$ & El Mostrador & Izquierda \\
\hline $\begin{array}{l}\text { Ley Antidiscriminación se resolverá en } \\
\text { comisión mixta }\end{array}$ & $\begin{array}{l}4 \mathrm{de} \\
\text { abril }\end{array}$ & La Nación & Centro \\
\hline
\end{tabular}




\begin{tabular}{|l|l|l|l|}
\hline $\begin{array}{l}\text { Jorge Reyes sobre Ley } \\
\text { Antidiscriminación: "Es una } \\
\text { parafernalia política" }\end{array}$ & $\begin{array}{l}9 \text { de } \\
\text { abril }\end{array}$ & El Mercurio & Derecha \\
\hline
\end{tabular}

\subsubsection{Procesamiento del corpus}

Tomando como unidad de análisis el complejo clausal, la pesquisa se basará en las herramientas proporcionadas por la TAV. Dicha propuesta tiene sus fundamentos teóricos en la Lingüística Sistémico Funcional y ha sido ampliamente estudiada, entre otros, por White (2004), Martin (2005), Martin y White (2005), Martin y Rose (2007) y Hood (2010). Se ocupa de estudiar y analizar "los recursos por medio de los cuales los hablantes/textos llegan a expresar, negociar y naturalizar determinadas posiciones intersubjetivas y en última instancia, ideológicas" (White 2004). En consecuencia, la TVA se preocupa de describir la construcción discursiva de la actitud y de la postura intersubjetiva. Los mecanismos discursivos son llamados recursos evaluativos y se dividen en tres sistemas semánticos: 1) ACTITUD, 2) GRADACIÓN y 3) COMPROMISO.

Según Kaplan (2004), en su traducción de Martin y White (2005), el sistema de ACTITUD hace referencia a los enunciados o elementos de los enunciados que transmiten una determinada evaluación positiva o negativa, o que puede interpretarse como una invitación al lector/oyente a suministrar sus propias evaluaciones. Dicho sistema está subdividido en tres regiones semánticas que se correlacionan con las dimensiones emotiva, ética y estética: 1) Afecto, recurso mediante el cual el hablante indica su disposición emocional o reporta respuestas emocionales de terceros hacia personas, cosas, situaciones o eventos, por ejemplo, (in)felicidad, (in) seguridad e (in)satisfacción; 2) Juicio, normas sobre cómo deben o no deben comportarse las personas, esto es, estima social (normalidad, capacidad y tenacidad) y sanción social (veracidad e integridad moral); y, 3) Apreciación, donde los sentimientos humanos hacia productos, procesos y entidades, se institucionalizan mediante reacciones (impacto y calidad), composiciones (balance y complejidad) y valuaciones.

La GRADACIÓN se refiere a los valores por medio de los cuales los hablantes gradúan el impacto interpersonal, es decir, aumentan o disminuyen la Fuerza de sus emisiones a través de intensificadores, amplificadores y enfáticos, y gradúan (desdibujan o agudizan) el Foco de sus categorizaciones semánticas, donde el valor representado podría tener un estatus central o prototípico, o, por el contario, un estatus marginal. Finalmente, el subsistema de COMPROMISO hace alusión a los recursos para posicionar la voz del hablante/autor en relación con las diversas proposiciones comunicadas por 
un texto; los significados por medio de los cuales los hablantes reconocen (heteroglosia) o ignoran (monoglosia) la diversidad de puntos de vista y por medio de las cuales negocian un espacio interpersonal para sus propias posiciones dentro de esa diversidad. En el caso de la heteroglosia, esta será extravocalizada, cuando es más explícita, y se subdivide en inserta (cita directa) o asimilada (cita indirecta), o intravocalizada, cuando es más implícita. Dentro de los principales recursos lexicogramaticales de COMPROMISO heteroglósico destacan la modalización, la polaridad afirmativa y negativa, la concesión, entre otros recursos que implican opciones del hablante o del escritor en el discurso (adaptado de Kaplan 2004 y White 2004).

\section{ANÁLISIS Y DISCUSIÓN}

Para el análisis se presentan algunos ejemplos representativos que describen el modo cómo tres medios de prensa nacional construyen la representación de las evaluaciones sobre el tema de la discriminación a propósito del caso Zamudio y la Ley Antidiscriminación ${ }^{6}$. En concreto, se pasará revisión a ciertas valoraciones asociadas a los objetos (Ley Antidiscriminación), a los actores sociales (Zamudio y otros que son valorados en este contexto) y a los eventos sociales (la golpiza a Zamudio y otros eventos que surgen vinculados con este). A este respecto, los postulados de Fairclough (1995), en relación con que el discurso implica tanto el texto como la práctica discursiva realizada por los hablantes en los textos y la práctica social donde práctica discursiva y texto son modelados ideológicamente, serán esenciales, pues nos permitirán "acceder" a cómo se producen los discursos y a cómo se vinculan unos con otros, esto es, mediante su convivencia, su reciclaje y su recontextualización, donde su producción, su circulación y su consumo contribuyen a la construcción de los contextos para dichos discursos.

6 En relación con la notación de los ejemplos, las valoraciones inscritas se presentan con negrita y subrayado; las valoraciones evocadas con cursiva; ambas pueden ser positivas $(+)$ o negativas (-); la Fuerza y el Foco se marcaron con mayúscula; se destacaron los eventos, se enmarcaron las valoraciones de polaridad negativa $\mathrm{y}$, en algunos casos relevantes, se enmarcaron con una línea punteada los casos donde se ve el uso de nosotros versus ellos. Al final de cada ejemplo se pone entre paréntesis el diario del que se extrajo dicho ejemplo, seguido de la fecha de aparición. 
Según la teoría del contexto de Fairclough $(1995,2008)$, consideramos necesario recalcar que en esta investigación el uso lingüístico (en nuestro caso el de los medios de comunicación) constituye una práctica social, que no solo es un modo de acción, sino que, además, se trata de un modo de acción situado histórica y socialmente, por tanto, las prácticas discursivas (en este caso, las crónicas noticiosas) están configuradas en términos sociales y, a su vez, son constitutivas de lo social. La propuesta de Fairclough también se centra en cómo las relaciones de poder restringen y controlan la productividad y la creatividad en las prácticas discursivas. Por otra parte, dicha propuesta se interesa en cómo una determinada configuración de prácticas discursivas relativamente estables, es decir, órdenes del discurso, constituyen un dominio hegemónico; el orden, en nuestro caso, podría corresponder a todas las noticias aparecidas en un mismo medio acerca de temas similares, dada la vinculación que ellas guardan entre sí, por el modo como ese medio comunica tal o cual noticia, a fin de conseguir tal o cual fin. Estas consideraciones serán abordadas en esta investigación, pues muy probablemente las tendencias ideológicas de los diarios seleccionados se verán reflejadas en las valoraciones que realicen respecto de la discriminación de género.

De acuerdo con lo anterior, presentamos en lo que sigue el Cuadro 2, de objetos, actores y eventos sociales que fueron valorados en los tres medios de prensa nacional.

Cuadro 2: Objetos, actores y eventos sociales valorados por cada diario

\begin{tabular}{|c|c|c|c|}
\hline & El Mercurio & La Nación & El Mostrador \\
\hline Objeto & $\begin{array}{l}\text { - Ley } \\
\text { Antidiscriminación }\end{array}$ & $\begin{array}{l}\text { - Ley } \\
\text { Antidiscriminación }\end{array}$ & $\begin{array}{l}\text { - Ley } \\
\text { Antidiscriminación }\end{array}$ \\
\hline $\begin{array}{l}\text { Actores } \\
\text { sociales }\end{array}$ & $\begin{array}{l}\text { Daniel Zamudio } \\
\text { Sociedad chilena } \\
\text { Jorge Reyes } \\
\text { (abogado ligado } \\
\text { a la UDI (Unión } \\
\text { Demócrata } \\
\text { Independiente) } \\
\text { Gobierno anterior } \\
\text { (Michelle Bachelet) } \\
\text { Gobierno actual } \\
\text { (Sebastián Piñera) } \\
\text { Organizaciones } \\
\text { sociales chilenas }\end{array}$ & $\begin{array}{l}\text { Daniel Zamudio } \\
\text { - } \text { Gobierno anterior } \\
\text { - } \text { Sociedad chilena } \\
\text { Reyes } \\
\text { Presidente de la } \\
\text { República } \\
\text { - Movilh } \\
\text { (Movimiento } \\
\text { de Integración } \\
\text { y Liberación } \\
\text { Homosexual) }\end{array}$ & 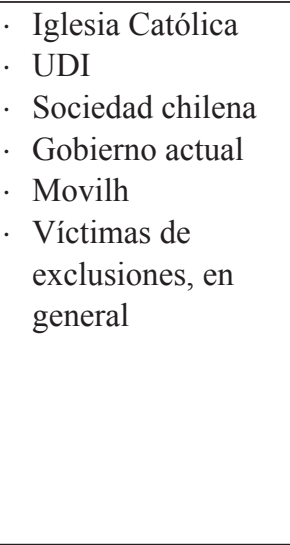 \\
\hline
\end{tabular}




\begin{tabular}{|c|c|c|c|}
\hline $\begin{array}{l}\text { Eventos } \\
\text { sociales }\end{array}$ & $\begin{array}{l}\text { - Golpiza a Zamudio } \\
\text {. Votación de la Ley } \\
\text {. Matrimonio } \\
\text { homosexual } \\
\text {. Aborto terapéutico }\end{array}$ & $\begin{array}{l}\text { Matrimonio } \\
\text { homosexual } \\
\text {. Votación de la Ley }\end{array}$ & $\begin{array}{ll}\text { - } & \text { Golpiza a Zamudio } \\
\text { - } & \text { Matrimonio } \\
\text { homosexual } \\
\text { - Discriminación, en } \\
\text { general }\end{array}$ \\
\hline
\end{tabular}

\subsection{Valoraciones en toRno a la Ley Antidiscriminación}

La Ley Antidiscriminación es valorada de distintas maneras en las noticias de los tres diarios que conforman el corpus de esta investigación. En relación con El Mercurio, en los dichos del ministro vocero de Gobierno, Andrés Chadwick (heteroglosia, extravocalización inserta), la Ley es valorada positivamente en el sentido de que no es la puerta al matrimonio homosexual, como se aprecia en el ejemplo 1, aunque, cabe destacar, no es tanto la Ley lo que se valora, sino el hecho de que no se abriría paso a un evento social que sí es valorado negativamente, como el matrimonio homosexual (polaridad negativa). De hecho, en los dichos del ministro siempre figura esta aclaración marcada por la Fuerza (está TOTALMENTE clarificado), y, como veremos más adelante, en relación con la Ley, es el mismo ministro quien incluye juicios negativos hacia la incapacidad del Gobierno anterior de darle curso al proyecto, implicando, a su vez, juicios positivos hacia el Gobierno que representa, que sí trabajaría en el proyecto.

(1) En cuanto a la posibilidad de que la ley permita la apertura para un matrimonio homosexual, el secretario de Estado dijo que "eso está TOTALMENTE clarificado y tampoco ha sido planteamiento ni petición de ninguna de las organizaciones. Sino que todas han comprendido de que el matrimonio en Chile entre un hombre y una mujer, es el matrimonio que hoy día nuestra legislación contempla y este proyecto no lo modifica EN NADA". (El Mercurio, 030412)

Por otro lado, la mencionada Ley aparece asociada fundamentalmente a los dichos de Reyes (heteroglosia), quien valora negativamente el proyecto de ley mediante apreciaciones inscritas que lo califican de "gran engaño", que "no aporta", "parafernalia política" y como una "seria pérdida" de derechos, como se observa en los ejemplos 2, 3 y 4 . En estas valoraciones negativas se emplea la Fuerza (GRAN engaño y SERIAS pérdidas) como recurso de GRADACIÓN, lo que permite enfatizar en lo negativo de la Ley, a juicio de Reyes. 
(2) “Es un GRAN engaño, no aporta a la ley que yaitenemos", dijo el abogado y ex asesor de la UDI, quien generó polémica por sus dichos sobre Daniel Zamudio. (El Mercurio, 090412)

(3) Como una "parafernalia política” calificó el abogado Jorge Reyes la tramitación de la Ley Antidiscriminación en el Congreso. El ex asesor del Servicio de Salud de la Región del Bíobio explicó que la medida legal sería un “engaño” y recordó el caso de la jueza Atala para explicar punto de vista. (El Mercurio, 090412)

(4) El abogado, reconocido por su lucha contra la píldora del día después, indicó que la gestión de la ley "es una parafernalia política para satisfacer a grupos minoritarios que LAMENTABLEMENTEinoS significarán a nosotros SERIAS pérdidas de derechos". (El Mercurio, 090412)

En La Nación, en tanto, se observan, apreciaciones negativas por manipulación de la Ley. Dichas valoraciones tienen voz en los dichos del Movilh (heteroglosia), como se desprende de los ejemplos 5 y 6 , actor social que no tiene cabida en las valoraciones hacia la Ley en El Mercurio. El Movilh, además, incluye apreciaciones y Fuerza ([proyecto] "cercenado" en los SIETE AÑOS) para valorar negativamente el accionar del Gobierno anterior y del Gobierno actual.

(5) La organización social, a través de un comunicado de prensa, aseguró que no apoyará el actual proyecto de ley debido a que éste fue "cercenado" en los SIETE AÑOS de discusión parlamentaria y terminó alejándose de su espíritu original. (La Nación, 030412)

(6) De hecho, 'acusañque "la versión actual del proyecto es incluso discriminatoria, por cuanto asocia a la diversidad sexual con delitos de connotación sexual". (La Nación, 030412)

En este sentido, por parte del vocero de Gobierno (heteroglosia) se producen apreciaciones positivas inscritas que vinculan el actuar del Gobierno actual en los avances del proyecto, intensificados mediante la Fuerza, tal como muestran los ejemplos 7, 8 y 9 donde, a su vez, se valora negativamente al Movilh ("quitó importancia" (7)).

(7) El vocero de La Moneda, Andrés Chadwick, defendió el actual proyecto y quitó importancia a_los reparos del movimiento, respondiéndoles' que "la ley no sólo va a proteger al Movilh". ( $L a$ Nación, $0 \overline{3} 0 \overline{4} 12$ ) 
A este respecto, también destacamos que en (5) y (7) la polaridad negativa es un recurso frecuente donde se reflejan las diferentes posturas de los actores implicados y sus valoraciones hacia la Ley.

En (8), desde la heteroglosia, se incluye una apreciación inscrita acerca de la clara voluntad del Presidente de la República por la aprobación del proyecto de ley, lo que da cuenta de la valoración positiva propia.

(8) donde el Ejecutivo apuesta a una PRONTA aprobación. ( $\mathrm{La}$ Nación, 030412)

En 9 se emplea un recurso de Foco (COMPLETAMENTE) insistiendo así en las soluciones que entrega el actual Gobierno.

(9) El ministro vocero de Gobierno, Andrés Chadwick, sostuvo que "hemos dado un paso MUY significativo, vamos en tierra COMPLETAMENTE derecha y estamos a pasos de tener un proyecto de ley que permita generar respeto y tolerancia por cada uno de los seres humanos que viven en nuestra patria, y luchar con MAYOR fuerza en el área de la educación, cultura y acción judicial contra toda forma de discriminación arbitraria". (La Nación, 040412)

La heteroglosia también está presente en los dichos del vocero de Gobierno en (10), donde se observa una clara valoración evocada mediante un juicio a la dirección adecuada del Presidente en el avance del proyecto de Ley.

(10) Explicó que los vetos "abarcan cuatro temas para mejorar $\mathbf{y}$ perfeccionar el provecto de lev", aclarando que éstos "no estaban sujetos a si la votación era una u otra, sino que corresponden a una convicción PROFUNDA del Presidente de cómo contribuir a potenciar el proyecto". (La Nación, 040412)

Por último, en El Mostrador, las valoraciones hacia la Ley Antidiscriminación se resumen en apreciaciones evocadas por parte de miembros de la Iglesia Católica (heteroglosia), quienes valoran negativamente la Ley por las implicancias que podría tener (como se aprecia en el ejemplo 11) y que, como vemos, no solo no lo hacen de manera explícita, sino que incluyen polaridad negativa para darle mayor relevancia a sus dichos $\mathrm{y}$, a su vez, evadir la referencia directa a la ley, pues no se habla de antidiscriminación, sino de "no discriminación"; en este sentido, se deja entrever que la aprobación de la Ley Antidiscriminación conllevaría, por ejemplo, al matrimonio homosexual o, bien, a la aceptación de la homosexualidad en la sociedad. 
(11) Las declaraciones del Movilh tuvieron lugar luego de las afirmaciones realizadas este viernes por monseñor Ricardo Ezzati, quien manifestó con respecto a la Ley Antidiscriminación que "hay que considerar bien qué cosa hay bajo del sombrero de la no discriminación”'. (El Mostrador, 310312)

Por otra parte, se incluyen los dichos del Movilh (heteroglosia) que, de manera contraria e inscrita, enjuician la manipulación de la Iglesia Católica y de la UDI, y valoran positiva y explícitamente la importancia de la Ley, como se desprende del ejemplo 12.

(12) Una "infundada campaña del terror" para impedir el avance de la ley antidiscriminación estaría realizando la Iglesia Católica y la UDI según el Movimiento de Integración y Liberación Homosexual (Movilh), el cual este sábado manifestó su rechazo a las declaraciones realizadas por personeros de ambas entidades e insistió en la "URGENTE necesidad para alcanzar justicia que tienen las víctimas de las exclusiones". ( $E l$ Mostrador, 310312).

Porúltimo, destacamos que en el caso de ElMostrador, la Ley Antidiscriminación no es tan valorada, como sí lo son las posturas contrarias entre las opiniones de los actores sociales, a saber, el Movilh, que solo tiene voz en La Nación y en El Mostrador; la Iglesia Católica, que solo es considerada en El Mostrador por oposición a las voces defensoras de la Ley, mostrando la polaridad entre defensores y detractores; y, la Unión Demócrata Independiente, cuyos representantes tienen amplia cabida en El Mercurio y que solo tienen voz en El Mostrador en la medida en que se les considera opositores a la Ley y a sus implicancias.

Sintetizando, podemos notar como en relación con la Ley las posturas de los diferentes medios reflejan claramente una diferente opción ideológica. En El Mercurio la valoración acerca de la Ley Antidiscriminación se centra en los temores de los ciudadanos hacia la aceptación social de la homosexualidad y lo que esta implicaría, la legalización del matrimonio homosexual, y deja en claro que un representante de la UDI puede fácilmente poner en entredicho la voz del Gobierno. El 3 de abril de 2012, el vocero de Gobierno descarta la posibilidad de que la Ley Antidiscriminación sea el primer paso para legalizar el matrimonio homosexual; sin embargo, el 9 de abril se vuelve a la carga, como si no se hubieran escuchando las palabras tranquilizadoras, recogiendo los dichos de Jorge Reyes acerca del riesgo o amenaza de una ley que -según sus palabras- busca satisfacer a grupos minoritarios. En consecuencia, se generaría una pérdida de derechos para el resto de los ciudadanos, que conforman la mayoría a la cual él se adscribe. 
Queda de manifiesto la soterrada lucha entre dos grupos irreconciliables, puesto que se descubre una idea maniqueísta: darle a la minoría un derecho involucra una pérdida, un retroceso para la mayoría, aunque se trate de un derecho humano. Ambas noticias revelan que el grupo dominante parte de la premisa (o el prejuicio) de que la sociedad chilena no acepta el matrimonio homosexual y que algunos tendrían interés por manipular la ley para legalizarlo.

EnLa Nación, por su parte, la valoración acerca de la Ley Antidiscriminación se centra en la posición del Gobierno y la tramitación de la Ley Antidiscriminación. El 3 de abril, aparece el vocero de Gobierno, en una noticia similar a la del mismo día en El Mercurio, defendiendo la ley, descartando que permita el matrimonio homosexual y respondiendo a las críticas del Movilh, pues se trata de una ley que no solo "los protege a ellos". El 4 de abril, La Nación se hace cargo de la tramitación de la ley en la Cámara de Diputados y del optimismo del Gobierno con respecto a su aprobación. La Nación aparece trasparentando la postura del gobierno que separa claramente la aprobación de la Ley Antidiscriminación de la defensa de los derechos homosexuales como se desprende de la frase "la ley no sólo va a proteger al Movilh".

Para finalizar, en El Mostrador, la valoración acerca de la Ley se centra en acusaciones y cuestionamientos hacia el Movilh, la glesia Católica y la UDI. La crónica del 31 de marzo muestra una ley desacreditada y cuestionada, fruto de una campaña del terror en su contra (como se aprecia en el ejemplo 12). La discusión de fondo - que ha sido suavizada en los otros medios analizados- tiene que ver con la discriminación hacia las minorías sexuales y el matrimonio homosexual.

\subsection{VALORACIONES EN TORNO A DANIEL ZAMUDIO Y OTROS ACTORES SOCIALES}

En relación con los actores sociales valorados en las crónicas noticiosas de los diarios de prensa nacional, en lo que sigue, presentaremos algunos ejemplos que nos permitirán dar cuenta de importantes diferencias en las valoraciones de los mismos. Asimismo, en esta sección incluiremos reflexiones sobre los dichos en los que se manifiestan implícita o explícitamente formas de discurso racista, a saber, la dicotomía nosotros versus ellos, mediante individualizaciones o identificaciones de actores sociales (van Leeuwen 1996).

En El Mercurio, debido a la cabida que se les otorga a actores sociales vinculados con el Gobierno actual, los dichos de Reyes (heteroglosia, 
extravocalización asimilada) juegan un rol importante en tanto constituyen juicios evocados a través de una sanción social hacia Zamudio y su comportamiento, como se aprecia en el ejemplo 13.

(13) Reyes generó una polémica la semana pasada, cuando dijo que la opinión pública tendría una postura diferente sobre la muerte de Zamudio si conociera MÁS antecedentes de su vida. (El Mercurio, 090312)

Dichos juicios, a su vez, se transforman en juicios a la integridad moral de Reyes, quien, implícitamente, adopta el rol de "polémico", aunque en este mismo diario se le otorga voz para dar una disculpa pública y señalar que fue mal interpretado, como puede observarse en el ejemplo 14.

(14) Tras la molestia que generaron sus dichos, el abogado dijo haber sido malinterpretado, argumento que reiteró en el programa de CHV, pues no tuvo la intención de atáacar la imagen del joven fálecidòtras una BRUTAL golpiza por presuntos neonazis. (El Mercurio, 090312)

En (14) observamos un juicio evocado positivo desde y hacia Reyes: "el abogado dijo haber sido malinterpretado, argumento que reiteró en el programa de $\mathrm{CHV}$, pues no tuvo la intención de atacar la imagen del joven fallecido tras una BRUTAL golpiza por presuntos neonazis". También en este diario, las valoraciones positivas mediante juicios de capacidad hacia el correcto actuar del Gobierno actual y hacia la mala gestión del Gobierno anterior son relevantes, gradadas, incluso, por la Fuerza, donde se deja ver el buen trabajo que el mismo Gobierno (a través de la voz de Chadwick, heteroglosia) considera que ha realizado, como se desprende del ejemplo 15.

(15) Consultado sobre la celeridad con que se ha visto la tramitación del proyecto de Ley Antidiscriminación desde la muerte del joven Daniel Zamudio (24), Chadwick sostuvo que "creo que eso no es justo, el Gobierno viene trabajando este proyecto de ley y $\underline{\text { lo ha reactivado }}$ POR COMPLETO desde hace va BASTANTE tiempo". (El Mercurio, 030312)

En La Nación, en tanto, se observan las primeras valoraciones de afecto asociadas con actores sociales, a saber, hacia Zamudio, en particular ("marcada por un minuto de silencio en homenaje...el fallecido joven"), mediante monoglosia, y hacia la sociedad, en general ("generar respeto y tolerancia...") con marcas de Fuerza de la voz de Chadwick (heteroglosia) (como se aprecia en los ejemplos 16 y 17). En (16), además, se observa un juicio hacia la sociedad intensificado por la Fuerza de la brutal golpiza, donde se deja entrever la intolerancia generalizada hacia la homosexualidad y se 
utiliza un recurso de valoración por orden marcado, al referirse al "fallecido joven". Por su parte, en (17), también es valorado positiva y explícitamente, a través de juicios, el Gobierno actual, por sus avances en el proyecto de Ley, lo que también se observó, entre otros, en (10).

(16) La sesión estuvo marcada por un minuto de silencio en homenaje a Daniel Zamudio, eêl joven homosexuali que perdió la vida tras ser BRUTALMENTE golpeado por una pandilla en el parque San Borja, y FUERTES cuestionamientos a los dichos del abogado Jorge Reyes hacia el fallecido joven. (La Nación, 040412)

(17) El ministro vocero de Gobierno, Andrés Chadwick, sostuvo que "hemos dado un paso MUY significativo, vamos en tierra COMPLETAMENTE derecha y estamos a pasos de tener un proyecto de ley que permita generar respeto y tolerancia por cada uno de los seres humanos que viven en nuestra patria, y luchar con MAYOR fuerza en el área de la educación, cultura y acción judicial. (La Nación, 040412)

Finalmente, en El Mostrador, el Movilh adquiere un rol protagónico cuando critica el actuar tanto de la UDI como de la Iglesia Católica mediante valoraciones inscritas negativas, intensificadas por la Fuerza "INFUNDADA campaña del terror", "NUEVAMENTE" (como se observa en los ejemplos $12,18$ y 19$)$.

(18) “(...) NUEVAMENTE la Iglesia Católica se está pronunciando contra el VALIOSO derecho humano de la no discriminación. ( $E l$ Mostrador, 310312)

(19) Crítico frente a la Unión Demócrata Independiente se mostró también el Movilh, al sostener que el partido rechaza el artículo 2 de la ley y asimismo "tiene el insensible descaro de afirmar que rechaza toda forma de discriminación". (El Mostrador, 310312)

En (12), además, se enjuicia a la sociedad que parece no aceptar la homosexualidad, para lo cual se emplea un recurso de nominalización ("víctimas de exclusiones" en lugar de "son excluidos"; "URGENTE necesidad para alcanzar justicia que tienen las víctimas de las exclusiones").

Junto con lo anterior, en El Mostrador aparece el juicio contrario, es decir, desde la Iglesia (en la voz de Ezzati, heteroglosia) hacia el Movilh, que es acusado de "aprovecharse del caso Zamudio", con lo que faltarían a la verdad (Ezzati emplea la polaridad negativa para evocar su juicio y lo intensifica con el recurso de Foco "DE VERDAD"), como se observa en los ejemplos 20 y 21. 
(20) “Aquí el que miente, DE VERDAD, no soy yo", exclamó el religioso. (El Mostrador, 040412)

(21) En entrevista en Radio Cooperativa, el religioso aseguró que el presidente del Movilh, Rolando Jiménez, difundió versiones sobre el actuar de la Iglesia Católica que no corresponden con la realidad. (El Mostrador, 040412)

Asimismo, Ezzati utiliza un recurso de afecto negativo que le permite graduar su valoración negativa hacia el Movilh ("lamento que"), lo modaliza con "sin duda alguna" y a ello suma el uso de polaridad negativa ("sin"), lo que refleja opciones, en este caso, tener o no tener las pruebas para una acusación, como se desprende del ejemplo 22.

(22) Lo que pasó acá es que, SIN DUDA ALGUNA, el Movilh ha querido aprovecharse de una situación que OBJETIVAMENTE no es COMO ellosila presentan, y lamento que una persona TAN pública pueda acusar de 'mentiroso' a otra persona sin tener los elementos objetivos para hacerlo. (El Mostrador, 040412)

Este mismo hecho, le permite a Ezzati valorar positivamente su actuar y, en su nombre, el de la Iglesia Católica, mediante el uso de juicios evocados, como se aprecia en el ejemplo 23.

(23) he actuado a través del vicario para la Familia, que acudió al hospital para darle la unción de los enfermos; (El Mostrador, 040412)

En síntesis, en El Mercurio se valora positivamente la preocupación permanente del Gobierno actual por tener una Ley Antidiscriminación, y negativamente a Daniel Zamudio. En La Nación, continúa la valoración positiva del Gobierno actual por su interés en votar la Ley y de afecto hacia Zamudio, lo que podría generar una solidaridad ideológica con los lectores. Por último, en El Mostrador, la Iglesia Católica y la UDI son valoradas negativamente por el Movilh, por no defender el derecho a la no discriminación y, en respuesta, monseñor Ezzatti devuelve la valoración negativa a Movilh, poniendo en duda la veracidad de sus dichos

\subsubsection{Nosotros versus Ellos}

En las valoraciones asociadas con los actores sociales puede observarse claramente la dicotomía nosotros versus ellos, a través del empleo de individualizaciones o identificaciones de actores sociales (van Leeuwen 1996). Bajo esta dicotomía se transparenta un prejuicio básico que tiene 
que ver con el hecho de identificarse con un grupo o sector social y no mezclarse con "otros", quienes manejan otros principios o valores. En el manejo de la "otredad", el grupo se hace fuerte, se reconoce a sí mismo y aparece amparado por el poder e incluso por los medios, asumiendo la primera persona (nosotros) y situando a la tercera persona (ellos) en una ignorada lejanía.

En las crónicas que nos ocupan, "la imagen del joven fallecido" (como se aprecia en el ejemplo 14) corresponde a una individualización realizada por Reyes (quien representa el nosotros), hacia Daniel Zamudio, donde están implicados ellos, es decir, los homosexuales y quienes los defienden y aceptan. Asimismo, en (16) se hace explícita la tendencia sexual de Zamudio, al dirigirse a él como, "el joven homosexual". Por otra parte, cuando en (4), Reyes utiliza el nosotros señalando: "LAMENTABLEMENTE nos significarán a nosotros SERIAS pérdidas", está planteando claramente su identificación con un sector de la sociedad opuesto a los grupos minoritarios cuyas demandas se busca satisfacer con la referida Ley. Lo mismo se observa en (22), donde el ellos corresponde al Movilh: "no es como ellos lo presentan" por oposición al nosotros, encabezado por el Gobierno y la Iglesia católica. "La ley no sólo los va a proteger a ellos", dice Chadwick, en La Nación, mostrando con claridad cuál es la postura del Gobierno frente a la discriminación, que abre su manto de protección para estar incluso con los que discriminan. Más casos concretos de esta dicotomía pueden verse en los ejemplos (2) (tenemos, nosotros), (3) (su punto de vista, que es el del Gobierno (nosotros)), (6) (acusan ellos, el Movilh), (7) (reprendiéndoles ellos) y (17) (hemos dado un paso).

Uno de los aspectos clave en este marco de referencias tiene que ver con el hecho de que es el prejuicio en el que se ancla esta idea de oposición. Recordemos que para Reyes, (como puede apreciarse en el ejemplo 13) la conducta de Daniel Zamudio no es intachable -como si eso fuese un argumento para no ir en su defensa en este caso-e inmediatamente agrega que "Al parecer hay una visión romántica respecto de lo que podría ser la tolerancia hacia los homosexuales, y prosigue poniendo como ejemplo que la propia familia de Zamudio lo había echado de la casa. Se trata, pues, de una visión romántica que claramente Reyes no comparte, dado que puede inferirse que para él los homosexuales son gente de vida desordenada. Por otro lado, Movilh también expresa sus juicios hacia la Iglesia Católica (como de observa en el ejemplo 18) al proponer que no es la primera vez que la Iglesia se pronuncia contra un derecho humano ("el VALIOSO derecho humano de la no discriminación"). El empleo de los deícticos -nosotros/ ellos- en las presentes crónicas permite aseverar que, claramente, se trata de dos posiciones frente al caso Zamudio y frente a la Ley Antidiscriminación, 
que no logran reconciliarse. Como es propio, nosotros representa el poder, las palabras del Gobierno, de la UDI y de la Iglesia; ellos, Zamudio, el Movilh y los discriminados.

\subsection{VALORACIONES EN TORNO AL EVENTO SOCIAL "GOLPIZA A ZAMUDIO" Y OTROS EVENTOS SURGIDOS EN ESTE CONTEXTO}

En relación con el evento social más relevante para efectos de esta investigación, la golpiza a Daniel Zamudio y los eventos asociados a este, queremos destacar lo siguiente: El Mercurio lo presenta asociado a la Fuerza, que califica la golpiza como BRUTAL (como se aprecia en el ejemplo 14), incluye valoraciones acerca de la votación de la Ley, donde destaca la voz de Chadwick (heteroglosia), que considera correcto el actuar del Gobierno en este proceso, como se observa en el ejemplo 24.

(24) Respecto a la posibilidad de que sea rechazada, Chadwick expresó que "si eso no se logra, desdramaticemos el tema. El Congreso funciona, iremos a la Comisión Mixta y se verá de qué manera se puede perfeccionar el proyecto". (El Mercurio, 030412)

Asimismo, establece valoraciones del matrimonio homosexual, enjuiciado negativamente (como puede verse en el ejemplo 1) y del aborto terapéutico, considerado como correcto porque permite salvar la vida de una madre, en la apreciación inscrita de Chadwick, como se desprende del ejemplo 25.

(25) "Y cuando se explica que el aborto terapéutico significa salvar la vida de la madre, cuando tiene un riesgo de salud, en perjuicio de la vida del niño. Eso se hace todos los días y un médico está obligado a hacerlo", apuntó Chadwick. (El Mercurio, 030412)

La Nación solo incorpora eventos asociados a la golpiza de Zamudio, como la votación de la Ley, en tanto proceso posiblemente polémico, como se desprende del ejemplo 26.

(26) Lo MÁS probable es que la votación no esté exenta de polémicas, pues el Movilh ya anunció su asistencia al Congreso. (La Nación, 030412)

Además, incorpora el matrimonio homosexual, también en los dichos de Chadwick, donde se lo valora negativamente si es que cambia el concepto de matrimonio, lo que se recalca mediante el uso de la polaridad negativa, como puede verse en el ejemplo 27. 
(27) Asimismo, el secretario de Estado recalcó que el proyecto no cambia las condiciones actuales del matrimonio, que es "entre un hombre y una mujer, es el que nuestra legislación contempla, y ésta ley no modifica en NADA, ni tampoco abre ninguna puerta porque señala que no es algo que puede argumentar en los tribunales". (La Nación, 030412)

Finalmente, El Mostrador considera la golpiza a Zamudio en el contexto de la discriminación en general, asociándola a la Fuerza, que califica la golpiza como BRUTAL y el periodo posterior como TRÁGICO (como se aprecia en los ejemplos 28 y 29), lo que implica juicios negativos que conllevan a la necesidad de justicia. En (28), asimismo, se destaca la nominalización "agresión homofóbica" que pone nombre a la acción, sentando precedente al permitir relacionarla con otros hechos del mismo tipo e incluso calificarla, como se hace en este caso, de "brutal". También se incluyen valoraciones hacia el matrimonio homosexual, pero solo como parte de las críticas a la UDI y a la Iglesia Católica.

(28) BRUTAL agresión homofóbica que sufrió el joven homosexual, que posteriormente le causó su muerte. (El Mostrador, 040412)

(29) Insistieron en la URGENTE necesidad de justicia para las víctimas de exclusiones. (El Mostrador, 040412)

En síntesis, todos los medios coinciden en señalar que se trató de una brutal golpiza o agresión a Daniel Zamudio. Cuando se trata de aludir al matrimonio homosexual todos los medios lo hacen para descartar que tenga alguna relación con el proyecto de ley antidiscriminación y, por último, en relación con la votación de la ley hay optimismo sin desconocer lo difícil que está resultando.

\section{CONCLUSIÓN}

En esta investigación intentamos realizar una indagación preliminar en torno a las valoraciones realizadas por tres medios de prensa nacional respecto del caso de Daniel Zamudio y la Ley Antidiscriminación. En este sentido, se propuso indagar acerca del modo cómo la prensa nacional, representada por El Mercurio, La Nación y El Mostrador, 'valoran' el tema de la discriminación de género a propósito del caso Zamudio y la Ley Antidiscriminación. La propuesta estuvo basada en la teoría del contexto 
de Fairclugh (1995), donde los textos constituyen prácticas discursivas que son modeladas ideológicamente en las prácticas sociales, y que, por tanto, construyen, reconstruyen, hacen y deshacen realidades sociales, en la medida en que su configuración es social $\mathrm{y}$, a su vez, contribuyen a configurar lo social.

A este respecto, el trabajo realizado nos permitió dar cuenta de la relevancia de las valoraciones en la prensa chilena, ya que, como vimos, las diferentes tendencias políticas de los diarios se ven reflejadas claramente en el espacio que le asignan a actores y eventos sociales, así como en los eventos sociales que incorporan sobre la base de la noticia de Zamudio y la Ley Antidiscriminación. No menos relevantes son las valoraciones asociadas a la Ley Antidiscriminación, donde quedaron de manifiesto no solo las ideologías de los medios de prensa nacional, sino que, además, los temores sociales y el prejuicio hacia las minorías sexuales. En consecuencia, El Mercurio se confirma como un medio de tendencia conservadora dirigido a un nivel sociocultural alto, preocupado por las decisiones que regulan la vida en sociedad; de hecho, en el análisis quedó de manifiesto cómo las voces que tienen cabida en este diario son, en gran medida, las de los representantes de la coalición política gobernante, que son, además, quienes se atribuyen la representación de los valores éticos y morales de la sociedad, que, de acuerdo con lo que manifiestan, estaría en contra de las minorías sexuales. La Nación se propone como el canal del gobierno, ocupado en valorar las acciones del Gobierno anterior y actual. Con respecto a los actores sociales que tienen voz en este medio, en el análisis se muestra al Gobierno y el Movilh. Por lo demás, este es el diario donde los enunciados monoglósicos tienen mayor presencia, ocupando una buena parte de ambas crónicas en la descripción y narración de lo que está aconteciendo con el debate acerca de la Ley en la Cámara de Diputados. Desde esta monoglosia se valoran positivamente las acciones del Gobierno actual y del Presidente de la República. Por último, El Mostrador se posiciona como un medio más combativo, donde se transmite sin censura la discusión entre los diferentes actores sociales, que tienen cabida en este medio. Así aparece con destacada relevancia la Iglesia Católica y el Movilh; dos actores que se invalidan mutuamente a través de descalificaciones que destruyen la base de confianza en lo que cada uno representa (la Iglesia Católica, por un lado, y el Movimiento de Liberación Homosexual, por el otro).

Junto con lo anterior, también fue de gran importancia analizar cómo se presentaron las identidades de un nosotros no homosexual y contrario a esta tendencia sexual, frente a un ellos homosexual y/o defensor de la homosexualidad. Como se desprende del análisis, resulta coherente observar -y confirma los hallazgos anteriores- que en El Mercurio y en La Nación 
predomina la voz del nosotros, a diferencia de lo que ocurre en El Mostrador, donde el ellos y sus defensores tuvieron mayor cabida. Esto se vincula con las ideologías que hay detrás de cada diario y con la forma como estas prácticas discursivas son modeladas para conseguir sus propósitos, cada uno (cada diario) con su visión acerca de cómo acercarse al tema de la discriminación de género, al caso Zamudio y a la Ley Antidiscriminación.

Tal como planteábamos en un principio, siguiendo los postulados de van Dijk, se trata aquí de una práctica social -el discurso de los medios de comunicación- cuyo poder es generalmente simbólico y persuasivo, cada medio se propone - en alguna medida e indirectamente- el control de las opiniones de sus lectores y para ello, se despliega una gama de recursos propios de los tres sistemas semánticos analizados, donde la elección de los actores sociales, la división o separación ideológica y semántica del ellos y el nosotros, sumados a la polémica y la descalificación, resultan ser la cara visible e invisible a veces, de un velado recurso de persuasión que, como ya hemos mencionado, funciona sobre la base de los supuestos temores y prejuicios de la sociedad.

En esta aproximación empírica al análisis de la Valoración, los sistemas de ACTITUD, GRADACIÓN y COMPROMISO han resultado especialmente útiles para el minucioso examen del tópico en las prácticas analizadas, confirmando que se trata de un potente aparato teórico y metodológico para caracterizar los recursos del nivel discursivo-semántico utilizados en estos medios. De hecho, no hubiese sido posible tener una imagen tan clara del modo como cada medio construye su realidad, si no nos hubiésemos adentrado en las apreciaciones valuativas (uno de los recursos más usados por los tres medios de prensa en el sistema ACTITUD), en los juicios de estima o sanción social, en la manera como los hablantes intensifican o disminuyen la fuerza de sus enunciados (GRADACIÓN) y en los recursos para posicionar la voz del hablante empleados en la prensa nacional (COMPROMISO).

Finalmente, cabe destacar que pese a que el corpus utilizado para la investigación fue bastante reducido, arrojó importantes datos para pensar en iniciar un estudio mayor, incluso en la diacronía y con herramientas como los grupos focales, donde, además, se podría constatar la relevancia de los datos arrojados en el análisis desde una perspectiva más centrada en la sociedad. Asimismo, sería de gran interés incluir otras perspectivas del análisis del contexto, como la de modelos mentales de van Dijk (2008b). De esta forma, el "acceso" a la producción de los discursos y a las relaciones que establecen unos con otros cobra mayor sentido, pues, como ya se mencionó, su producción, su circulación y su consumo contribuyen a la construcción de sus contextos. En definitiva, tanto la perspectiva de análisis empleada, como las mencionadas recientemente, podrían ofrecer importantes constructos 
teóricos y metodológicos, que nos permitan contribuir de una manera eficaz a los estudios sobre la discriminación de género, dada la relevancia que reviste este tema en nuestra sociedad.

\section{REFERENCIAS BIBLIOGRÁFICAS}

Collado, Carolina y David Jofré. 2010. Discriminación de género en la legitimación de liderazgos públicos. Análisis crítico del discurso periodístico del diario La Nación en los años 2000 y 2006. Revista F@ro - Tesis, Año 6, Número 11. Disponible en: http://web.upla.cl/revistafaro/

De la Fuente, Mario. 2004. Análisis crítico del discurso y racismo en los medios de comunicación, en M. Villayandre (ed.). Actas del V Congreso de Lingüística General (León, marzo de 2002), Madrid: Arco Libros, pp. 1047-1057.

Fairclugh, Norman. 1995. Critical Discourse Analysis: the Critical Study of Language. London: Longman.

2008. El análisis crítico del discurso y la mercantilización del discurso público: Las universidades. Discurso \& Sociedad 2(1): 170-185. Traducción de Elsa Ghio.

Foucault, Michael. 1992[1970]. El orden del discurso. Traducción de Alberto González Troyano. Buenos Aires: Tusquets editores.

Hood, Susan. 2010. Appraising Research: Evaluation in Academic Writing. London: Palgrave Macmillan.

Kaplan, Nora. 2004. Nuevos desarrollos en el estudio de la evaluación en el lenguaje: la teoría de la valoración. Boletín de Lingüística 22: $52-78$ [en línea]. Disponible en: redalyc. uaemex.mx/redalyc/pdf/347/34702203.pdf [Consulta: 20/05/2012]

Latorre, Guillermo y Vega, Olly. 2003. Del dicterio al encomio. Estrategias semánticas generales y la imagen de las superpotencias en El Siglo de Chile (1957-1962). Estudios Filológicos 38: 31-47.

MARTIN, JAMES. 2005. Invocación de actitudes: el juego de la gradación de la valoración en el discurso. Revista Signos 38 (58): 195-220.

Martin, James y David Rose. 2007. Working with Discourse. Meaning beyond the clause. Londres, Nueva York: Continuum.

Martin, James y Peter White. 2005. The Language of Evaluation. Appraisal in English. New York: Palgrave Macmillan.

Merino, María Eugenia. 2007. El discurso de la discriminación percibida en Mapuches de Chile. Discurso \& Sociedad 1(4): 604-622

Merino, María Eugenia y Mauricio Pilleux. 2003. El uso de estrategias semánticas globales y locales en el discurso de los chilenos no mapuches de la ciudad de Temuco. Estudios Filológicos 38: 111-119.

Merino, María Eugenia, Quilaqueo, Daniel y José Luis Saiz. 2008. Una tipología del discurso de discriminación percibida en mapuches de Chile. Revista Signos 41 (67): 279-297.

Merino, María Eugenia y Cristian Tileaga. 2011. La construcción de identidad de minorías étnicas: un enfoque discursivo psicológico a la autodefinición étnica en acción. Discurso \& sociedad Vol. 5(3): 569-594 (Traducido por José Silva Garcés).

Molero, Lourdes y Julián CABeZA. 2007. "El enfoque semántico-pragmático en el análisis del discurso: teoría, método y práctica". En Análisis del Discurso. A. Bolívar (ed.). 2007, pp. 201-26. 
Oteíza, Teresa. 2006. El discurso pedagógico de la historia. Santiago: Frasis.

2006. Reseña de Working with Discourse. Meaning beyond the clause de James Martin y David Rose. 2003. London, New York: Continuum. Estudios Filológicos 41: 293-299.

Oteíza, Teresa y Claudio Pinuer. 2012. Prosodia valorativa: construcción de eventos y procesos en el discurso de la historia. Discurso \& sociedad, Vol. 6(2).

Pilleux, Mauricio y María Eugenia Merino. 2004. El prejuicio étnico desde una perspectiva del análisis del discurso. Onomázein 9: 169-186.

Pino, Ana Patricia y María Eugenia Merino. 2010. Discriminación e identidad étnica en el discurso oral de adolescentes mapuches en contexto escolar de la ciudad de Temuco. Discurso \& Sociedad, Vol. 4(1): 103-119.

Rodríguez-Bailón, Rosa y Miguel Moya. 2003. La diferencia percibida en valores como proceso vinculado a las relaciones intergrupales de payos y gitanos. Psicothema 15(2): 176-182.

SOLER, SANDRA. 2004. Pensar la relación análisis crítico del discurso y educación. El caso de la representación de indígenas y afrodescendientes en los manuales escolares de ciencias sociales en Colombia. Discurso \& Sociedad 2(3): 642-678.

Soto, Guillermo. 2011. Reseña de Discurso y Poder de Teun van Dijk. Gedisa. 2009. Discurso \& Sociedad, Vol. 5 (2): 432-441.

Traverso-YéPez, Martha. 2005. Discursos Racistas: Institucionalización del Racismo a través de las Prácticas Lingüísticas. Revista Interamericana de Psicología/Interamerican Journal of Psychology, Vol. 39, $\mathrm{N}^{\circ} 1:$ 61-70.

van Dijk, Teun. 1988. El discurso y la reproducción del racismo. Lenguaje en contexto 1 (1 y 2): 131-180).

1994. Discurso, Poder y Cognición Social Conferencias. Cuadernos N², Año

2. Maestría en Lingüística. Escuela de Ciencia del Lenguaje y Literaturas. Disponible en: http://www.discursos.org/Art/Discurso,\%20poder\%20y\%20cognici\%F3n\%20social.pdf 1996. Análisis del discurso ideológico. Trad. De R. Alvarado. UAM-México: $15-43$.

Gedisa.

2003a. Ideología y discurso. Una introducción multidisciplinar. Barcelona:

2003b. Racismo y discurso de las élite. Barcelona: Gedisa.

2004a. Discurso y dominación. Grandes conferencias en la facultad de ciencias humanas. Bogotá: Universidad Nacional de Colombia.

2004b. Critical Discourse Analysis and Context. Ponencia en la First Annual International Conference of Critical Discourse Analysis. Valencia, España. (comp.). 2007. Racismo y discurso en América Latina. Barcelona: Gedisa.

2008a. Semántica del Discurso e Ideología. Trad. De Cristina Perales. Revista Discurso\&Sociedad, Vol 2(1): 201-261.

2008b. Context and discourse. En Discourse and context. A sociocognitive Approach. New York: Cambridge University Press.

2009. Discurso y poder. Contribuciones a los estudios críticos del discurso. Barcelona: Gedisa.

van LeEuwen, Theo. 1996. Text and Practices. Carmen Caldas-Coulthard y Malcolm Coulthard. Londres y Nueva York : Routledge.

White, Peter. 2004. Un recorrido por la teoría de la valoración [en línea]. Disponible en: www.grammatics.com/appraisal/SpanishTranslation-AppraisalOutline.pdf [Consulta: 20/05/2012] 
Wodak, Ruth. 2003. De qué trata el análisis crítico del discurso. Resumen de su historia, sus conceptos fundamentales y sus desarrollos. En Wodak, Ruth y Michael Meyer, Métodos de análisis crítico del discurso. Barcelona: Gedisa. 